\title{
Identification of a G2-like transcription factor, OsPHL3, functions as a negative regulator of flowering in rice by co- expression and reverse genetic analysis
}

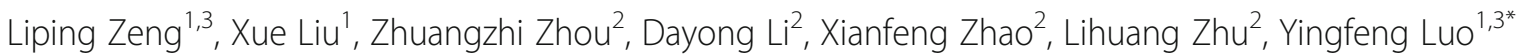 \\ and Songnian $\mathrm{Hu}^{1,3^{*}}$
}

\begin{abstract}
Background: Flowering time is a key trait for regional adaption and seed production in rice (Oryza sativa L.). Forward and reverse genetic studies have characterized a number of flowering-time genes. However, co-expression analysis has not been used to identify the flowering-time genes.

Results: We predicted a G2-like family transcription factor, OSPHL3, by co-expression networks analysis with photoperiodic flowering pathway genes. OsPHL3 contains a MYB-CC domain, and was localized in the nucleus with transcriptional activation potential. OSPHL3 was mainly expressed in the leaves and exhibited a circadian rhythmic expression pattern. Rice lines overexpressing OSPHL3 showed a delayed flowering time in the genetic background of TP309 under both long-day (Beijing) and short-day (Hainan) conditions. By contrast, the knockout rice lines of OsPHL3 by CRISPR/Cas9 technology promoted flowering time regardless of genetic backgrounds (i.e. Nipponbare and TP309) or day length. Further analysis indicated that OsPHL3 delayed flowering time by down-regulating the expression of Hd3a and RFT1 through promoting Hd1 under long-day conditions (LDs), or suppressing Ehd1/Hd1 under short-day conditions (SDs).
\end{abstract}

Conclusions: Our results suggested that co-expression analysis is a useful strategy for identifying novel floweringtime genes in rice.

Keywords: Co-expression, Flowering time, OsPHL3, MYB-CC transcription factor, G2-like, Rice

\section{Background}

Flowering time is one of the most important agronomic traits in determining grain yield and regional adaptation [1-3]. In rice, there are two independent pathways controlling photoperiodic flowering. The first one is OsGI-Hd1-Hd3a pathway [4-6], which is conserved between Arabidopsis thaliana and rice [7]. The second one is the Ghd7-Ehd1-Hd3a/RFT1 pathway, which is unique in rice [6, 8-10]. The florigen proteins, $H d 3 a$ and RFT1, work as integrated mobile flowering signals, and move

\footnotetext{
* Correspondence: luoyf@big.ac.cn; husn@big.ac.cn

${ }^{1}$ CAS Key Laboratory of Genome Sciences and Information, Beijing Institute of Genomics, Chinese Academy of Sciences, NO.1 Beichen West Road, Chaoyang District, Beijing 100101, China

Full list of author information is available at the end of the article
}

from leaf to the shoot apical meristem to induce reproductive development $[11,12]$.

The rice OsGI-Hd1-Hd3a pathway has a one to one correspondence with Arabidopsis GIGANTEA (GI) - CONSTANS (CO) - FLOWERING LOCUS T (FT) [7]. OsGI promotes the expression of $H d 1$, but $H d 1$ has different roles under LDs and SDs [5]. Under short day conditions (SDs), $H d 1$ activates $H d 3 a$ expression, while under long day conditions (LDs), it represses $H d 3 a$ expression [6, 7]. The functional conversion of $H d 1$ is determined by interacting with the phytochrome $B(p h y B)$-mediated signaling [13]. For the Ghd7-Ehd1-Hd3a/RFT1 pathway that is unique to rice, Grain number, plant height, and heading date 7 (Ghd7) acts as upstream of Early heading date1 (Ehd1), which does not have an ortholog in Arabidopsis.

(c) The Author(s). 2018 Open Access This article is distributed under the terms of the Creative Commons Attribution 4.0 International License (http://creativecommons.org/licenses/by/4.0/), which permits unrestricted use, distribution, and 
Under SDs, Ehd1 expression is induced by the products of Ehd2 [14], Ehd3 [15], Ehd4 [16] and OsMADS51 [17], but repressed by OsCOL4 [18]. Under LDs, Ehd1 expression is upregulated by Ehd2 [14], Ehd4 [16] and OsMADS51 [17], downregulated by OsCOL4 [18], OsCOL10 [19] and DTH8 [20].

Until now, most of the flowering-time genes were identified by map-based cloning in mutant plants or plants with natural variations, or alternatively by overexpression and knockdown transgenic plants [1]. For example, the function of OsMADS34 [21] and OsTrx1 [22] was investigated by knockdown transgenic lines; while that of OsMADS15 [23], OsMADS50 [24, 25], OsMADS56 [25], OsMYB1R1 [26], OsBBX14 [27] and OsCOL13 [28] was revealed by overexpression transgenic lines. Co-expression analysis in rice has been proven to be a useful tool for identifying the function of transcription factors, such as $H Y R$ in regulating the photosynthesis process and RSR1 in controlling starch biosynthesis $[29,30]$. However, the co-expression analysis has not been used to predict the flowering-time genes in rice.

Here, we conducted co-expression networks analysis in rice to interrogate genes that can play roles in flowering-time. Our study identified a G2-like family transcription factor containing Myb-CC domain that we referred to as OsPHL3. Functional characterization revealed that OsPHL3 plays an important role in regulating rice flowering time.

\section{Results}

Gene co-expression analysis identified G2-like family transcription factors involving in flowering time pathway in rice

In plants, leaves perceive information of day-length and temperature changes, which was subsequently used to determine flowering time [31, 32]. The mobile flower-promoting signal molecules, Hd3a and RFT1, are generated in leaves and transferred to the shoot apical meristem to induce flowering $[11,12]$. We used a published leaf transcriptome data [33] to predict flowering time regulators in rice.

The key rice flowering time genes, including OsGI, Ghd7, Hd1, Ehd1, RFT1, Hd3a, OsMADS14 and OsMADS15, were selected as "guide genes" to identify the co-expressed genes using leaf transcriptome data.

Among the candidate genes involved in flowering time regulation, we were particularly interested in transcription factors, because they accounted for $82 \%$ of known flowering-time genes [1].We calculated absolute value of the Pearson correlation coefficient and selected those greater than 0.8 between the flowering time regulators and TFs (Additional file 1: Table S1). We illustrated the top five most abundant TF families with member genes of co-expression to flowering time regulators (Additional file 1: Table S1). Among these TFs, the shared top-ranked co-expressed TFs were the member of G2-like family (Additional file 1: Table S1; Additional file 2: Figure S1).

With the absolute value of Pearson correlation coefficient greater than 0.6, 28 G2-like family transcription factors were co-expressed with flowering time pathway genes (Fig. 1). Of those genes, 21 genes were positively co-expressed and 7 genes were negatively co-expressed. Negative regulation of flowering-time genes increase grain yield of rice $[9,26,35,36]$. Therefore, to identify negative regulators of flowering time are of agricultural importance. We were intrigued by one of the identified TFs, LOC_Os09g12750, which displays negatively correlated co-expression with many flowering regulators including OsGI, Ghd7, Hd3a and OsMADS14. These results led us to the hypothesis that LOC_Os09g12750 was a new negative regulator of flowering time. We chose LOC_Os09g12750 for further studies, and referred to it as OsPHL3 (see below).

\section{Features of OsPHL3}

According to the Rice Genome Annotation Project Database (http://rice.plantbiology.msu.edu), LOC_Os09g12750 encodes a G2-like family transcription factor with a length of 250 amino acids and a molecular mass of approximately $27 \mathrm{KD}$. The pfam database shows that the protein has one Myb DNA-binding domain (35-86) and one CC domain (127-174) (http://pfam.xfam.org/search/sequence). As revealed by phylogenetic analysis of homologous proteins between rice and Arabidopsis, LOC_Os09g12750 is closely related to AT3G24120 (AtPHL3, PHR1 like3) (Fig. 2a). Protein sequence alignment of OsPHL3 and AtPHL3 showed that they were highly conserved in the Myb and $\mathrm{CC}$ domains (Fig. 2b). We hereby named LOC_Os09g12750 as OsPHL3.

\section{Expression pattern of OsPHL3}

We used quantitative real-time PCR (qRT-PCR) to determine the expression level of OsPHL3 mRNA in various rice tissues and organs. The results showed that OsPHL3 was ubiquitously expressed in root, culm, leaf, young panicle and mature panicle. The expression level of OsPHL3 was highest in leaves in both rice varieties of Nipponbare and TP309 (Additional file 3: Figure S2).

Interestingly, we detected a diurnal expression pattern of OsPHL3 by quantifying the relative abundance of its expression in young leaves in the rice varieties of TP309. The OsPHL3 transcript was much more abundant at dawn and midnight under both controlled long day (CLD) and controlled short day (CSD) conditions 


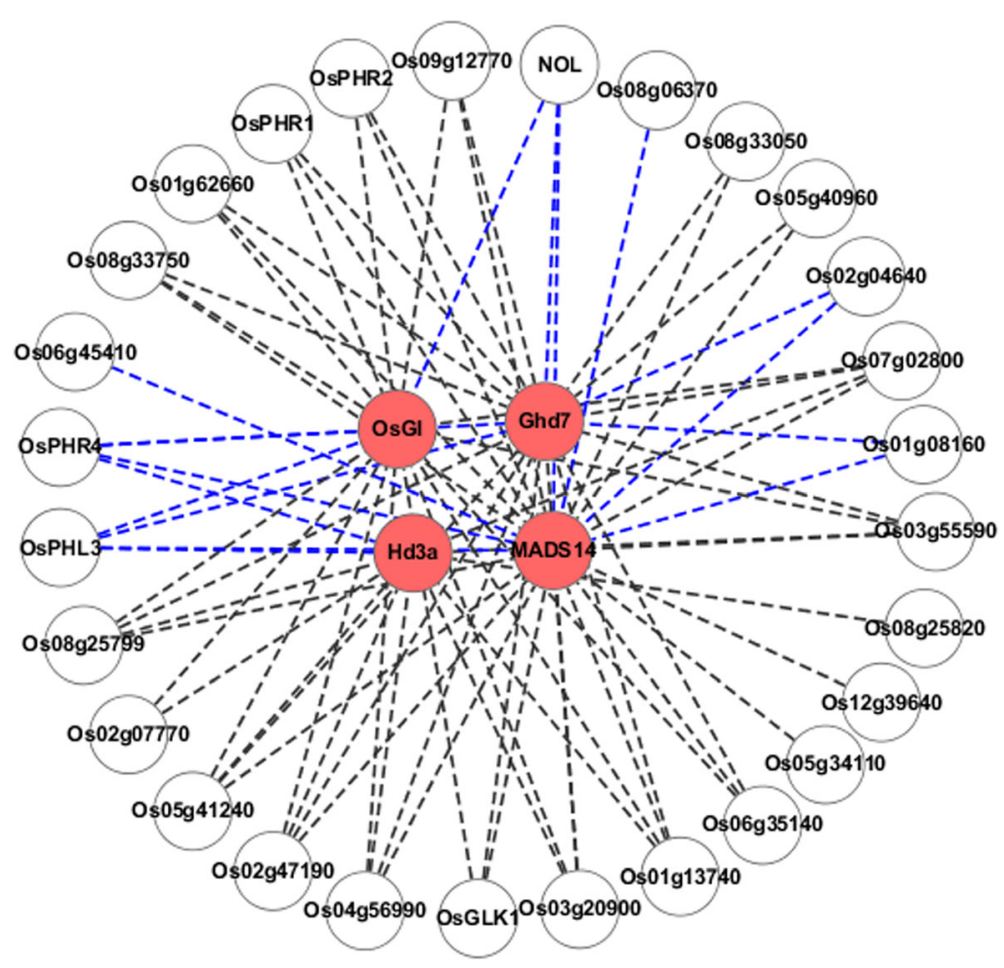

Fig. 1 Co-expression networks of rice G2-like transcription factors and flowering time regulators. Co-expression network between flowering time regulators and G2-like transcription factors based on a Pearson correlation coefficient cutoff of 0.6. Nodes in the maps represent genes. Red node represents the flowering- time genes in photoperiodic pathway. The blue lines connecting two genes represent transcription factors negatively co-expressed with photoperiodic flowering pathway genes. Black lines connecting two genes represent transcription factors positively coexpressed with photoperiodic flowering pathway genes

(Additional file 3: Figure S2), suggesting that OsPHL3 was expressed in a diurnal manner in rice.

\section{OsPHL3 is localized in the nucleus and has transcriptional activation potential}

We assayed the subcellular localization of OsPHL3. We fused the OsPHL3 with green fluorescent protein (GFP) driving by the cauliflower mosaic virus $35 \mathrm{~S}$ promoter. The OsPHL3 protein localized predominantly in the nucleus (Fig. 3a-d), suggesting that OsPHL3 likely conducts nuclear function.

To examine if OsPHL3 acts as transcriptional activator, we fused full and partial coding region of OsPHL3 with GAL4 DNA binding domain and transformed into yeast. The results showed that the BD-OsPHL3 (1-251) and BD-C terminal (101-251) fusion protein had transcriptional activation. We speculated that OsPHL3 had the potential to activate transcription in yeast, and that $\mathrm{CC}$ domain was required for transcriptional activation (Fig. 3e).

The OsPHL3-OE delayed rice flowering time under LD and SD conditions

To determine whether OsPHL3 is involved in the regulation of flowering time, we placed full-length coding region of $O S P H L 3$ into the constitutive cauliflower mosaic virus $35 \mathrm{~S}$ (CaMV35S) promoter driven over-expression vector and transformed the constructs into the rice variety TP309 by Agrobacterium. We obtained 12 independent T0 transgenic OsPHL3 overexpression (OsPHL3-OE) plants. In $\mathrm{T} 1$ families, the transgene-positive plants flowered later than the transgene-negative plants. Two overexpression homozygous lines (OE-4 and OE-6) from $\mathrm{T} 2$ progeny had a higher expression level of OsPHL3 in leaves and delayed flowering time (Fig. 4a-b). On average, OsPHL3-OE transgenic plants showed a significantly delayed flowering time than WT controls, either by 16 days under natural long day conditions (NLDs, Beijing), or 12 days under natural short day conditions (NSDs, Hainan) (Fig. 4c). These findings support our hypothesis that OsPHL3 is a negative regulator of flowering time.

\section{Knockout of OSPHL3 caused early flowering in long-day and short-day conditions}

In order to further validate the function of OsPHL3 in flowering time regulation, osphl3 mutants were constructed using the CRISPR/Cas9 technology. We selected two unique sequences in CDS region (36-54 nt and 92-111 nt) as targets for Cas9 cleavage (Fig. 5a), 
A

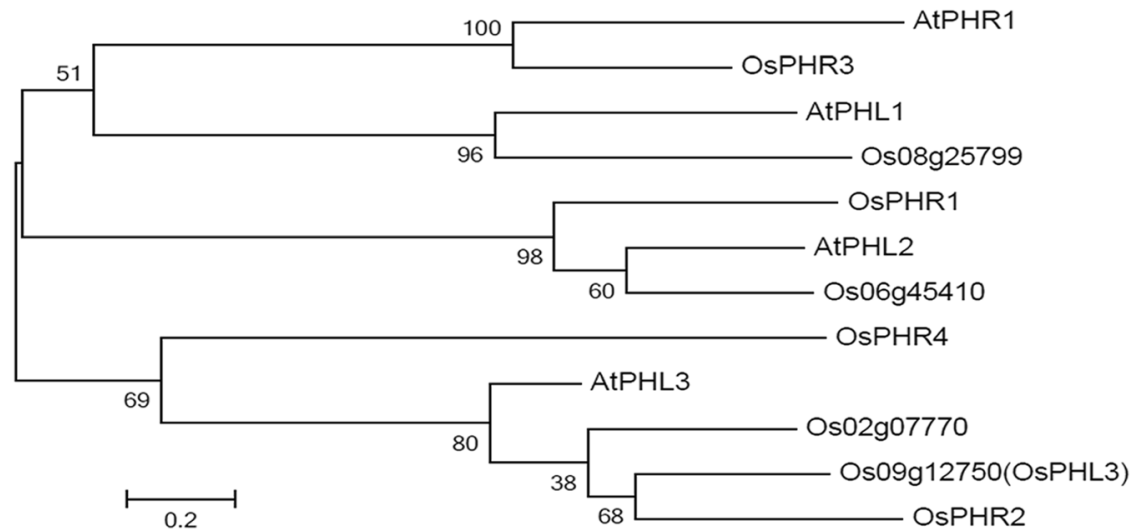

B

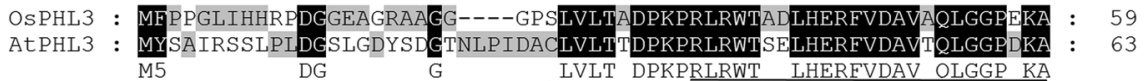

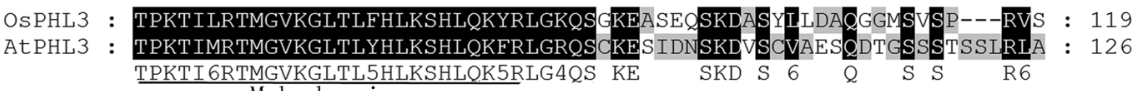
Myb domain

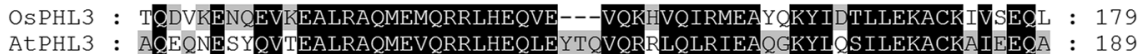

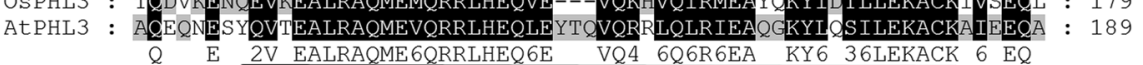
CC domain

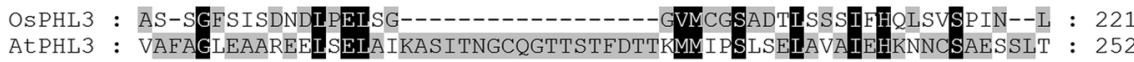
AtPHL $3:$ VAFAGLEAARELSEIAIKASITNGCQGTTSTFDTTKMMIISLSEIAVAIEHKNNCSAESSLT : 252

OSPHL3 : HSPEGKPTPSGIEG-----------OMILQKSPELKRKSC--- : 250 AtPHL $3:$ SSTVGSPVAALMKKRQRGVFGNGDSVVVGHDAGWVMPSSSIG : 295

Fig. 2 OsPHL3 is closely homologous to AtPHL3 in Arabidopsis thaliana and share a conserved MYB-CC domain. a Phylogeny of OsPHL3 and related MYB-CC proteins between rice and Arabidopsis. An un-rooted tree was constructed by the sequences of MYB DNA-binding domain and CC domain. b Protein sequence alignment between OsPHL3 and AtPHL3. The conserved Myb DNA-binding domain (35-86) and CC domain (127-174) were underlined

and generated multiple mutant lines. Two homozygous knockout lines of OsPHL3 (OsPHL3-CRISPR-3 and OsPHL3-CRISPR-5) were obtained from TP309 genetic background. OsPHL3-CRISPR-3 contains a Cytosine insertion at the position of 52 bp of CDS and OsPHL3-CRISPR-5 has a 2-bp deletion beginning at the position of $107 \mathrm{bp}$. We also created osphl3 mutants using the same method in Nipponbare, a day-length-sensitive cultivar, and successfully obtained two homozygous knockout lines (OsPHL3-CRISPR-2 and OsPHL3-CRISPR-11). Of these, OsPHL3-CRISPR-2 contains an inserted Thymidine at the position of 107 bp of CDS and OsPHL3-CRISPR-11 contains an inserted Cytosine at the position of $109 \mathrm{bp}$.

Under NLDs (Beijing), osphl3 mutants of TP309 promoted flowering by 21 days than WT on average, while under NSDs (Hainan) osphl3 knockout lines promoted flowering by 18 days than WT on average. The knockout lines of OsPHL3 of Nipponbare promoted flowering by 26 days than WT on average under NLDs (Beijing), while promoted flowering by 13 days than WT on average under NSDs (Hainan) (Fig. 5b-e).

Taken together, under both NLD (Beijing) and NSD (Hainan) conditions, knockout lines of OsPHL3 displayed an early-flowering phenotype in TP309 and Nipponbare. These data strongly support that OsPHL3 acts as a negative regulator of flowering time.

Expression of photoperiodic flowering pathway genes in the OsPHL3-OE plants under LD and SD conditions

To investigate the functional mechanism of OsPHL3 in photoperiod flowering pathway, we compared the expression pattern of six flower time genes (OsGI, Ghd7, Hd1, Ehd1, RFT1 and Hd3a) in OsPHL3-OE and WT control under CLD and CSD conditions. 

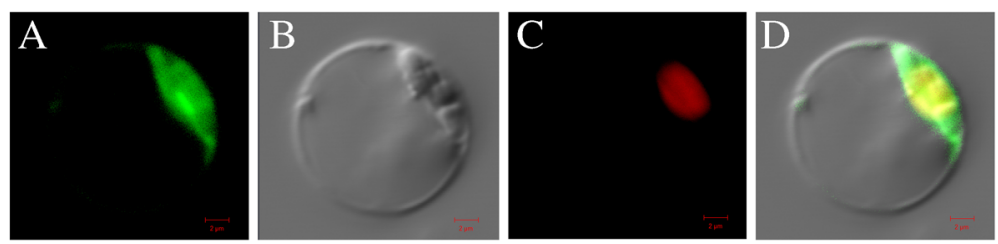

$\mathrm{E}$

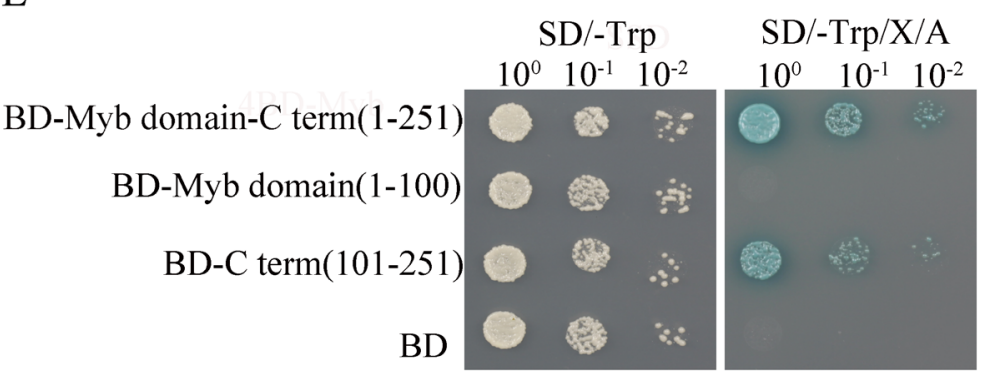

Fig. 3 Subcellular localization and transactivation analysis of OsPHL3. a Subcellular localization of OsPHL3-GFP fusion protein under the control of CaMV35S promoter. b Bright field. c The nuclear marker mCherry-VirD2NLS vector (mCherry). d Merged image. Bar =2um from A to D. (E) OsPHL3 full-length coding region, N-terminal region containing Myb-DNA binding domain (1-100 amino acids) or the C-terminal region containing CC binding domain (101-251 amino acids) were individually inserted into pBD-GAL4 DNA binding-domain contained plasmid. The empty pBD vector was used as control. The expression of OSPHL3 coding region and OSPHL3-C terminal showed resistance to toxic drug Aureobasidin A

In rice, the florigen genes, $H d 3 a$ and $R F T 1$, are crucial regulators of the transition from vegetative to reproductive phase. Thus, we firstly investigated the expression level of Hd3a and RFT1 in WT (TP309) and OsPH$L 3-O E$ lines. For all time points, the expression levels of $H d 3 a$ and RFT1 in WT control were much higher than in OsPHL3-OE lines under CLD and CSD conditions (Fig. 6). This result indicated that OsPHL3 functioned in the upstream of $H d 3 a$ and RFT1.

Two other genes, $H d 1$ and Ehd1, are responsible to integrate a network of genes to control the expression levels of florigen genes, $\mathrm{Hd} 3 \mathrm{a}$ and RFT1, and to control flowering time. Therefore, to detect whether OsPHL3 regulates $H d 3 a$ and RFT1 though $H d 1$ and $E h d 1$, we examined the expression levels of Hd1 and Ehd1 in OsPHL3-OE lines and WT (TP309). Under controlled long day conditions (CLDs), Hd1, which functions as a negative regulator of florigen under LDs, was expressed at a higher level in OsPHL3-OE lines than in WT control in all sampled time points, while Ehd1 didn't exhibit consistent expression manner (Fig. 6a). Under controlled short day conditions (CSDs), the expression of Ehd1 and $H d 1$ were constitutively repressed in the $O s P H-$ L3-OE lines compared with WT (Fig. 6b). As controls, the expression level of GI and Ghd7 were also tested, but we found no difference of them between OsPHL3-OE and control (TP309) (Additional file 4: Figure S3).

Taken together, the late-flowering phenotype of $\mathrm{OsPH}$ L3-OE plants may be at least partially caused by the down-regulation of Hd3a and RFT1 expression. Under SDs this was achieved through repression of Ehd1/Hd1, whereas under LDs it was generated by promotion of Hd1 (Fig. 7).

\section{Discussion}

Gene co-expression networks can be useful for identifying flowering time genes

Gene co-expression analysis is based on the assumption that genes with similar expression pattern may be functionally related [34]. This approach has been used for predicting the function of uncharacterized genes involved in many biological processes in plants, such as Arabidopsis glucosinolate biosynthesis [35], wheat spike architecture regulation [36], the tomato fruit ripening [37] and soybean oil synthesis [38]. In rice, gene co-expression networks successfully predicted the function of RSR1 (Rice Starch Regulator1) in starch biosynthesis [30] and HYR (HIGHER YIELD RICE) in photosynthesis process [29]. In this study, we identified a flowering time regulator OsPHL3 by gene co-expression analysis. Our results revealed that gene co-expression analysis was a powerful tool for predicting gene function from transcriptome data.

\section{G2-like family transcription factors are also involved in the regulation of rice flowering}

Golden2-like (G2-like) are members of GARP superfamily transcription factors [39]. OsPHL3 belongs to G2-like family transcription factor containing MYB-CC domain. In rice, the G2-like transcription factors participate in 

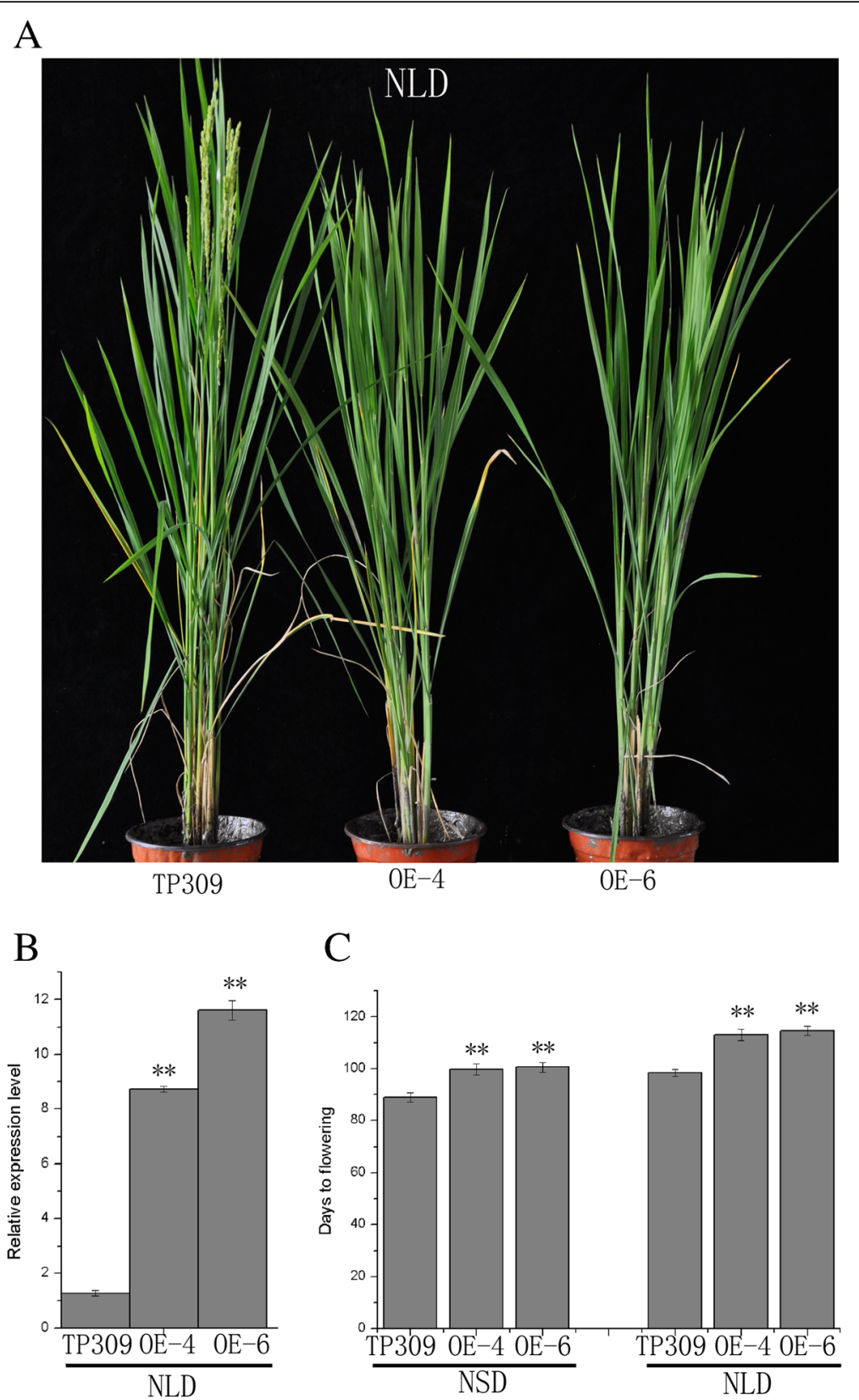

$\mathrm{C}$

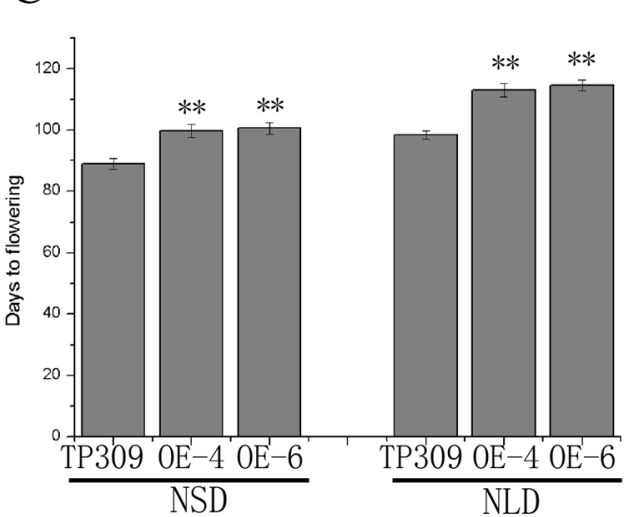

Fig. 4 OsPHL3 overexpression lines delayed flowering. a Phenotype characteristics of WT and OsPHL3-OE, 107-day-old plants, generated from TP309 genetic background under natural long day (NLD; Beijing). b The expression level of WT control and OsPHL3-OE (the overexpression lines 4 and 6). c Flowering time of WT control and OsPHL3-OE under NLDs (Beijing) and NSDs (Hainan) by Student's t test (** $p<0.01$ )

chloroplast formation, nutrient assimilation, as well as stress and disease resistance. One of the G2-like members $O s G L K$ is a key regulator of chloroplast development and also plays a role in resistance to pathogen invasion [37]. Another G2-like factor SLL1 regulates leaf rolling [38]. Additionally, the NIGT1 is a nitrate-responsive regulator [39]. Moreover, the OsPHR1, OsPHR2, OsPHR3 and OsPHR4 participate in Pi-starvation signaling [33, 34, 40]. Our current study reveals that G2-like transcription factor also plays a critical role in regulating flowering time in rice.
OsPHL3 acts as a novel repressor of flowering under LDs and SDs

Currently, the cloned floral genes could be divided into two categories: day-length independent genes and day-length dependent genes. The first group is the constitutive flowering regulators regardless of day-length [8, 14-18, 25, 27, $28]$. The second group encompasses genes that are functionally associated with day-length [9, 20, 22, 40-44].

Our current work identified OsPHL3 as a constitutive repressor of flowering under both LDs and SDs. The 


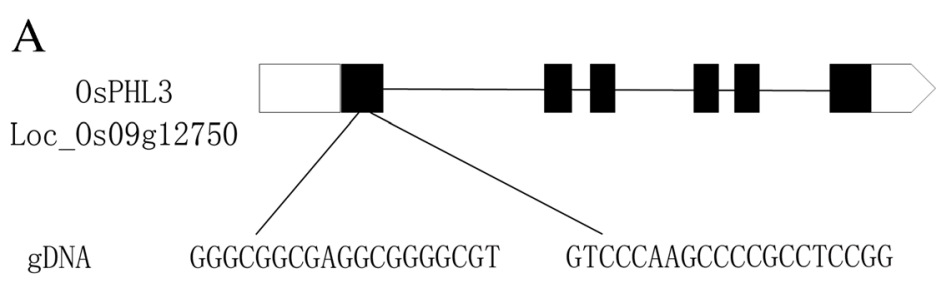

$\mathrm{B}$

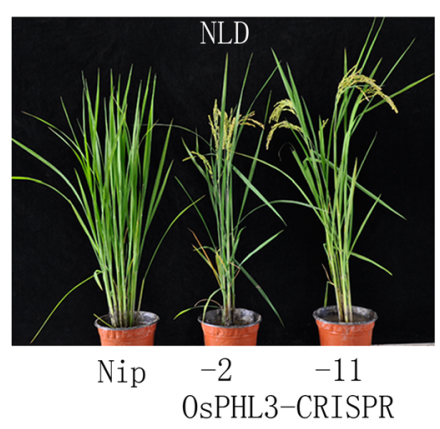

$\mathrm{C}$

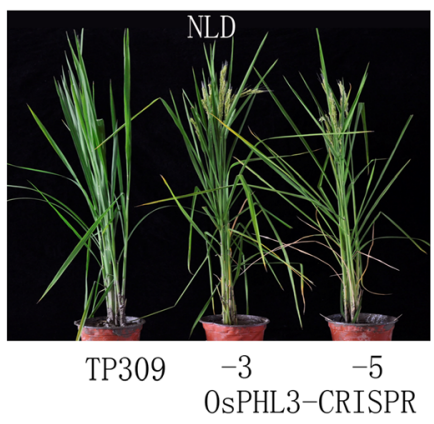

$\mathrm{D}$

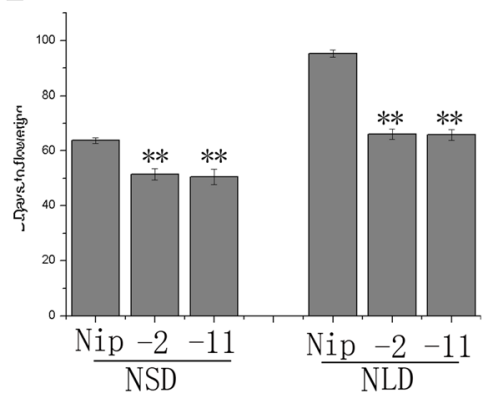

$\mathrm{E}$

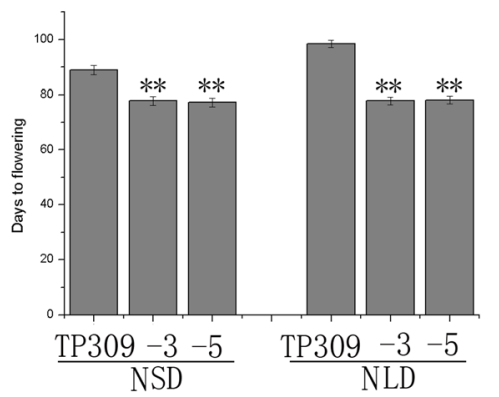

Fig. 5 The phenotypic characterization of OsPHL3 knockout lines. a Two target sites for knocking out OsPHL3 by the CRISPR/Cas9 system. b Phenotype of osph/3 mutants, 78-day-old plants, generated from Nipponbare genetic background under natural long day (NLD; Beijing). c Phenotype of osph/3 mutants, 86-day-old plants, generated from TP309 genetic background under natural long day (NLD; Beijing). d Statistical data for flowering time of Nipponbare and osph/3 mutants under NLDs (Beijing) and NSDs (Hainan). e Statistical data for flowering time (**p $<0.01$; Student's $t$ test) of TP309 and osph/3 mutants under NLDs (Beijing) and NSDs (Hainan)

OsPHL3-OE transgenic plants displayed a late-flowering phenotype with the genetic background of TP309, whereas the knockout mutants exhibit an early-flowering phenotype under two different day length conditions and two genetic backgrounds. Therefore, these results support that the function of OsPHL3 was highly conserved between rice cultivars TP309 and Nipponbare.

In rice, there are two pathways to control the flowering time: one is the conserved OsGI-Hd1-Hd3a pathway and the other is the rice unique Ghd7-Ehd1-Hd3a/RFT1 pathway. The diurnal expression pattern indicated that OsPHL3 functions as a negative flowering regulator by inhibiting the expression of RFT1 and Hd3a. This was achieved through promoting the expression of $\mathrm{Hd} 1$ under LDs, and through suppressing the expression of Ehd1/Hd1 under SDs (Fig. 7). Thus, our data suggest that OsPHL3 is involved in Ehd1- and Hd1-mediated regulatory pathway in rice. Whether OsPHL3 directly regulated Ehd1, Hd1 and other factors remains to be determined. Future work will aim to dissect the cis-regulatory elements directly bound by OsPHL3 on chromatin, and to identify functionally partner proteins interacting with OSPHL3.

\section{Conclusions}

First, gene co-expression networks allowed us to identify OsPHL3, a new gene involved in regulating the flowering pathway in rice. Second, overexpression of OsPHL3 delayed flowering time in rice under $\mathrm{LD}$ and SD conditions. Knockout of OsPHL3 by genome-editing tool CRISPR/Cas9 resulted in earlier flowering regardless of day-length. Third, OsPHL3 repressed flowering time by impacting Ehd1/Hd1-mediated pathway under SDs, and Hd1-mediated pathway under LDs. Our study indicates 

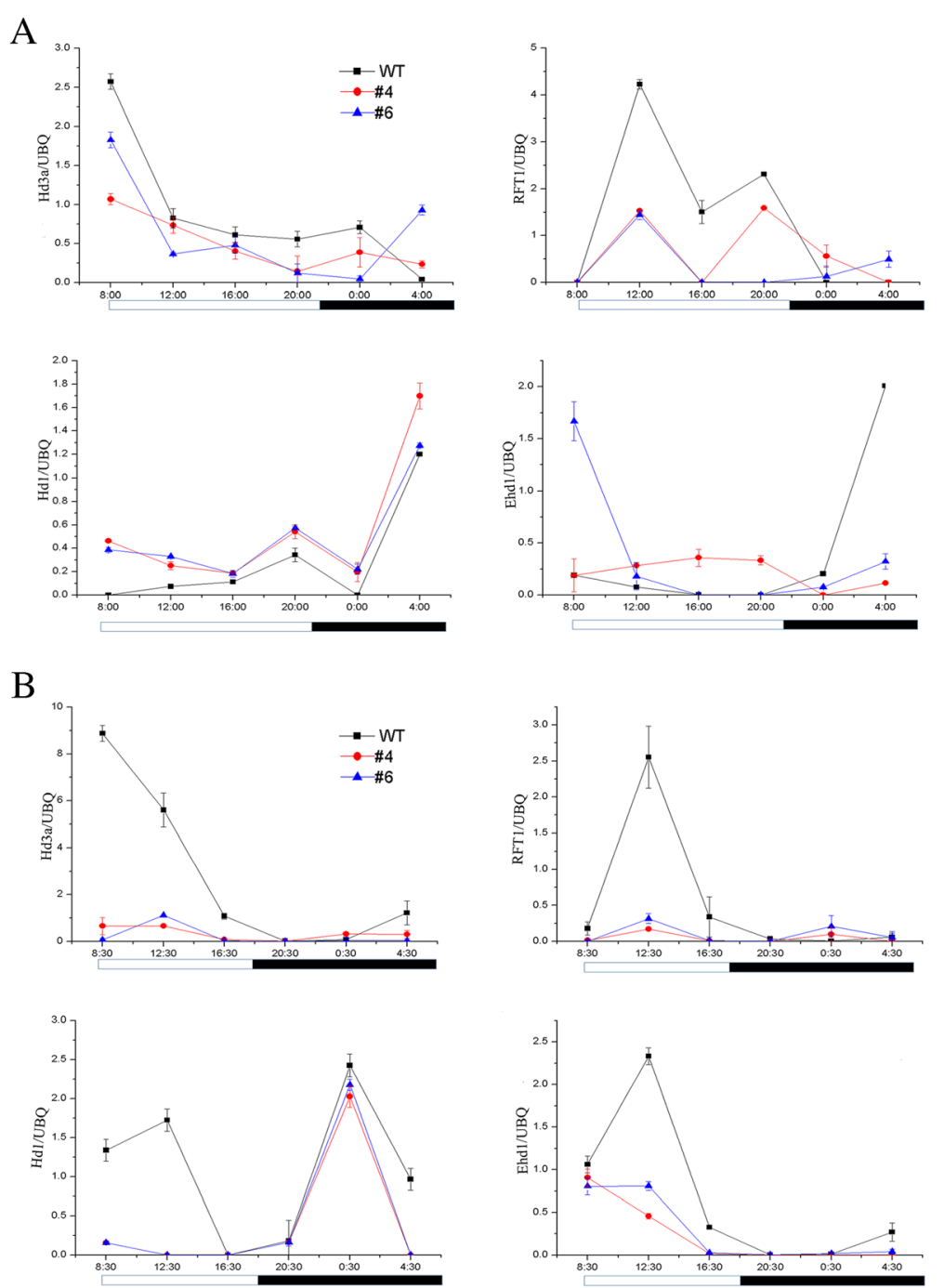

Fig. 6 Diurnal expression pattern of key floral regulators between WT and OsPHL3-OE transgenic plants. a-b Real time PCR revealed the transcript levels of Hd3a, RFT1, Hdl and Ehd1 under LDs (a) and SDs (b). Ubiquitin gene of rice was used as internal control. The black lines represent the WT. Red and blue lines represent OsPHL3-OE4 and OsPHL3-OE6 transgenic plants, respectively. White bars indicate the light period, and black bars indicate the dark periods. The numbers below the bars represent the hours of the day. The mean of each point is based on the average of three technical repeats and two biological repeats

that gene co-expression network analysis is a powerful tool for identifying genes affecting flowering time in rice.

\section{Methods}

\section{Plant materials and growth conditions}

Japonica rice (Oryza sativa L.) variety Nipponbare and Taipei309 (TP309) were used in this study. Nipponbare is the day length-sensitive variety. All rice plants were grown in the field of Institute of Genetics and Developmental Biology, Chinese Academy of Sciences, in Hainan $\left(18^{\circ} 30^{\prime} \mathrm{N}, 110^{\circ} 2^{\prime} \mathrm{E}\right)$ and Beijing $\left(40^{\circ} 13^{\prime} \mathrm{N}, 116^{\circ} 13^{\prime} \mathrm{E}\right)$.

The rice plants for diurnal expression analysis were firstly sown in pots in the greenhouse. After growing under natural day-length conditions for 20 and 50 days, the rice plants grown in a controlled-growth chamber under controlled long day (CLD) ( $14 \mathrm{~h}$ light/10 h darkness) and controlled short day conditions (CSD) (10 h light/14 h darkness) conditions with light intensity of 800 umol. $\mathrm{m}^{-2} \mathrm{~s}^{-1}$ for additional 14 days. Leaf samples were collected from 34-day-old plants under CLDs and 64-day-old plants under CSDs. We collected leaves under CLDs and CSDs seedlings in $4 \mathrm{~h}$ interval for total RNA extraction.

\section{Bioinformatics analysis}

The transcriptome data was downloaded from the website (https://www.ncbi.nlm.nih.gov/geo/query/acc.cgi?acc=GSE 54274) (GSE54274_rice_leaf_section_fpkm.txt.gz) [33]. The 


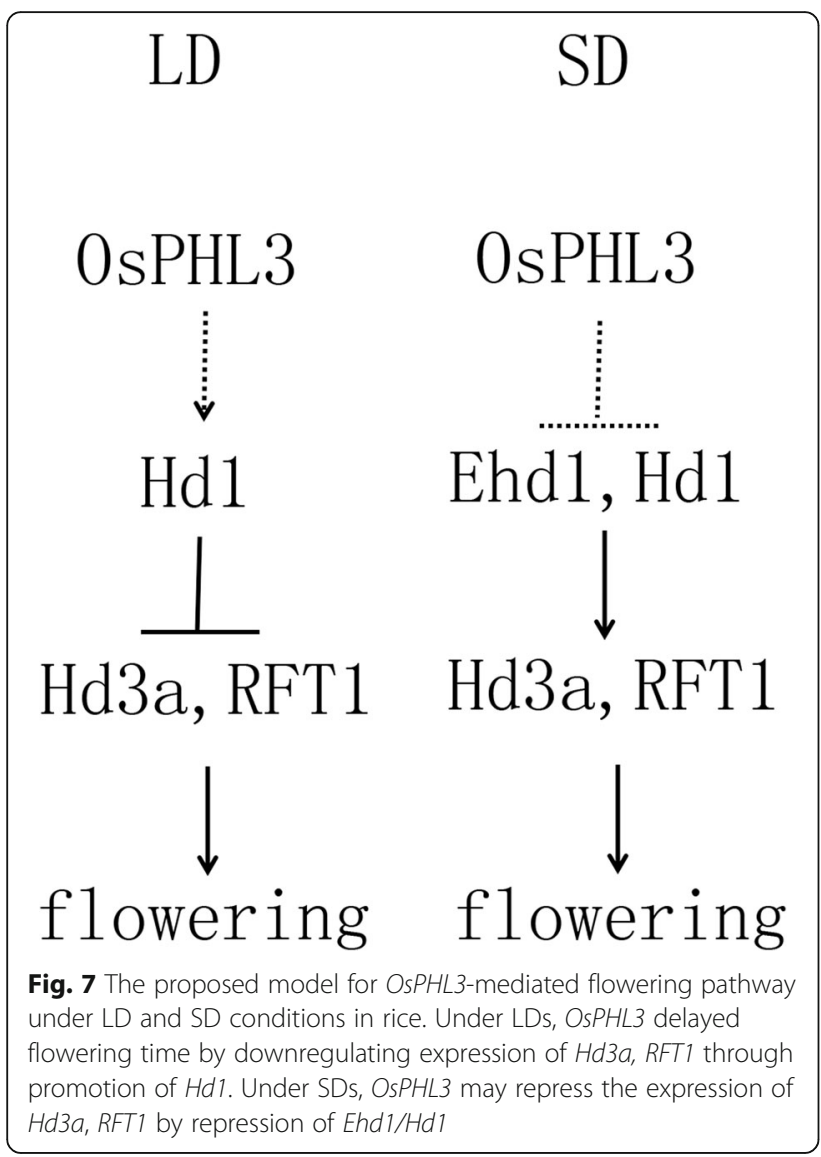

14-day-old rice leaves were cut into 11 parts, which represented the immature-to mature gradient of variation in gene expression $[33,45]$. The family information of rice transcription factors were downloaded from Planttfdb (http:// planttfdb.cbi.pku.edu.cn/) [46]. We calculated absolute value of the Pearson correlation coefficient between flowering time regulators and TFs using custom Perl script. The absolute value of the Pearson correlation coefficient greater than 0.8 between the flowering time regulators and TFs were listed in Additional file 1: Table S1. With the absolute value of Pearson correlation coefficient greater than 0.6, 28 G2-like family transcription factors co-expressed with flowering time genes (Fig. 1), which were constructed by Cytoscape v.2.8.3 [47]. Venn diagram was created using the online tool (http://bioinformatics.psb.ugent.be/webtools/Venn/).

All rice G2-like protein sequence were downloaded from Rice Genome Annotation Project (http://rice.plantbiology.msu.edu/); the Arabidopsis protein sequences of four G2-like sequences were downloaded from The Arabidopsis Information Resource (TAIR) (http://www.arabidopsis.org/). The alignment between rice and Arabidopsis was generated with ClustalW [48] using default setting (http://clustalw.ddbj.nig.ac.jp/). We used the software GeneDoc to perform the graphical output between OsPHL3 and AtPHL3 alignment. The neighbor-joining trees were constructed using MEGA software [49]. Domain prediction was carried out by Pfam database [50] (http://pfam.xfam.org/search/sequence).

\section{Vector construction and transformation}

To construct the overexpression vector, the 750-bp coding region of OsPHL3 (LOC_Os09g12750) was amplified using KOD plus DNA polymerase (Toyobo, Japan). Gene specific primers, 5'-GAATTC ATGTTCCCGCCTGG GCTGATCCACCACCGGCC-3'.

5'-GGATCC CCGCAAGACTTGCGCTTAAGCTCA GGTGACTT- 3' (EcoR I and BamH I sites underlined) were used to amplify the coding sequence of OsPHL3 using cDNA of Nipponbare as template. The digested PCR amplicons were cloned into the vector pEZR (K)-LN and confirmed by Sanger sequencing. The construct was introduced into the Agrobacterium tumefaciens LBA4404 by electroporation and subsequently transformed into the rice cultivar TP309 [51].

Positive transgenic plants were detected through the amplification of GFP in the vector and another fragment of OsPHL3 using the forward primer OsPHL3-F 5' - CCCG AGAAAGCAACACCTAAAA-3' and reverse primer GFP-R 5' - GTCGTCCTTGAAGAAGATGGTGC -3'. PCR amplification was carried out using the following profile: $95^{\circ} \mathrm{C}$ for 5 min; followed by 32 cycles at $95^{\circ} \mathrm{C}$ for $20 \mathrm{~s} ; 58{ }^{\circ} \mathrm{C}$ for $30 \mathrm{~s}$, $72{ }^{\circ} \mathrm{C}$ for $40 \mathrm{~s}$; and final extension at $72{ }^{\circ} \mathrm{C}$ for $7 \mathrm{~min}$.

We used CRISPR/Cas9 system to generate osphl3 plants by the Biogle Company (Hangzhou, China) [52]. Two sgRNAs targeting OsPHL3 CDS: sg37: 5' -GGGCGGCGA GGCGGGGCGT-3', and sg93: 5' -GTCCCAAGCCCCGC CTCCGG-3'. Vector pBGK032 containing single sgRNA was introduced into rice cultivar TP309 and Nipponbare by $A$. tumefaciens strain EHA105. Each constructs obtained more than 14 independent transgenic lines. To confirm the successful mutants, the genome DNA was extracted from the T2 generation transgenic plants to amplify the target regions and Sanger sequencing. The primers used were listed in Additional file 5: Table S2.

\section{Subcellular localization of OsPHL3 protein}

To detect the subcellular localization of OsPHL3 protein, full-length CDS of OsPHL3 was fused to green fluorescent protein (GFP) driving by the $35 \mathrm{~S}$ CaMV promoter. The mCherry-VirD2NLS vector (mCherry) was used as a nuclei marker. The protoplasts were isolated from 14-d-old rice seedlings and transformed with OsPHL3-pEZR (K)-LN. Fluorescence in the transformed protoplasts were visualized using confocal microscope (Zeiss Axio Imager.Z2) [53].

\section{Analysis of transcriptional activation}

We used Yeastmaker Yeast Transformation System 2 (Clontech, USA) to analysis transcriptional activation 
potential. The full-length coding sequence, the $\mathrm{N}$-terminal and $\mathrm{C}$-terminal deletion were amplified with the primers (Additional file 5: Table S2) by PCR and cloned into pGBKT7 to fuse the GAL4 binding domain to create the plasmids BD-Myb domain-C term (1-251), BD-Myb domain (1-100) and BD-C term (101-251). The transformants were grown on -Trp SD media or Trp/-His/-Ade SD media.

\section{RNA isolation and quantitative real-time PCR analysis}

Total RNA was isolated from different tissues and organs using Trizol reagent (Invitrogen, USA). DNA digestion was treated with DNase (Takara, Japan), and first-strand cDNA was synthesized by SuperScript kit (Takara, Japan). The rice ubiquitin gene was used as an internal control and quantitative RT-PCR was performed using the Bio-Rad CFX96 real-time system. The procedure was: $95^{\circ} \mathrm{Cfor} 3 \mathrm{~min}$ and 42 cycles of $95{ }^{\circ} \mathrm{C}$ for $5 \mathrm{~s}, 60{ }^{\circ} \mathrm{C}$ for $10 \mathrm{~s}$. The primers of photoperiodic flowering pathway genes (OsGI, Hd1, Hd3a, RFT1, Ehd1, Ghd7) were obtained as reported previously $[54,55]$. Average values were obtained from three technical replicates and two biological replicates. The primers were listed in Additional file 5: Table S2.

\section{Additional files}

Additional file 1: Table S1. The Pearson correlation coefficient value between flowering time regulators (OsGl, Ghd7, OsMADS14 and Hd3a) and top-five transcription factor families. (XLSX $19 \mathrm{~kb}$ )

Additional file 2: Figure S1. Venn diagram illustrating shared and unique top-five transcription factor families between OsGl, Ghd7, OsMADS14 and Hd3a. (TIF $2261 \mathrm{~kb}$ )

Additional file 3: Figure S2. (A) Expression analysis of OsPHL3 in various tissues and organs in the variety of TP309 and Nipponbare. (B) Diurnal rhythm pattern of OSPHL3 expression under CLDs in the variety of TP309. (C) Diurnal rhythm pattern of OsPHL3 expression under CSDs in the variety of TP309. (TIF $58 \mathrm{~kb}$ )

Additional file 4: Figure S3. Diurnal expression patterns of OsGl and Ghd7 in WT and OsPHL3-OE in the genetic background of TP309 under CLDs and CSDs by qRT-PCR analysis. (TIF $107 \mathrm{~kb}$ )

Additional file 5: Table S2. The sequence of primers used in this study. (DOCX $13 \mathrm{~kb}$ )

\section{Abbreviations}

CLD: controlled long-day; CLDs: controlled long-day conditions; CO: CONSTANS; CSD: controlled short-day; CSDs: controlled short-day conditions; DTH8: Days to heading 8; Ehd1: Early heading date 1; FT: FLOWERING LOCUS T; Ghd7: Grain number, plant height and heading date 7; Gl: GIGANTEA; Hd1: Heading date 1; Hd3a: Heading date 3a; LD: Long-day; LDs: Long-day conditions; OsPHL3-OE: OSPHL3-overexpression; RFT1: RICE FLOWERING LOCUS T 1; SD: Short-day; SDs: Short-day conditions; WT: Wild-type

\section{Acknowledgements}

We would like to thank Xinyu Tan, Jixiang Wang and Xiaochun Bi (Beijing Institute of Genomics, Chinese Academy of Sciences) for the preparation of rice genomic DNA. We thank Qi Wu (College of Pharmacy and Biological Engineering, Chengdu University), Dedong Yin (Reproductive Physiology laboratory, National Research Institute for Family Planning), Lili Hao and Shuangyang Wu (Beijing Institute of Genomics, Chinese Academy of Sciences) for helpful discussions.

\section{Funding}

This work was supported by Strategic Priority Research Program of the Chinese Academy of Sciences (XDA08020102), the National Natural Science Foundation of China (31000561 and 31771473), and the Youth Innovation Promotion Association of Chinese Academy of Science (2017140). The funding agencies did not participate in the design of the study, the collection, analysis, and interpretation of data, or in writing the manuscript.

\section{Availability of data and materials}

The sequence used in this study can be found in The Arabidopsis Information Resource (TAIR, http://www.arabidopsis.org/) and the Rice Genome Annotation Project (http://rice.plantbiology.msu.edu/).

\section{Authors' contributions}

SNH, YFL designed the research. LPZ carried out most of the experiments and drafted the manuscript. YFL and LPZ analyzed the data. XL and ZZZ participated in the protoplast transformation and transcriptional activity assay. XFZ participated in phenotype analysis. LHZ, DYL, XL, ZZZ and XFZ discussed the results and commented on the manuscript. All authors have read and approved the final manuscript.

\section{Ethics approval and consent to participate}

The experimental research on plants, including collection of plant material, was complied with the institutional, national, or international guidelines. And field studies were conducted in accordance with local legislation.

\section{Consent for publication}

No applicable.

\section{Competing interests}

The authors declare that they have no competing interests.

\section{Publisher's Note}

Springer Nature remains neutral with regard to jurisdictional claims in published maps and institutional affiliations.

\section{Author details}

${ }^{1}$ CAS Key Laboratory of Genome Sciences and Information, Beijing Institute of Genomics, Chinese Academy of Sciences, NO.1 Beichen West Road, Chaoyang District, Beijing 100101, China. ${ }^{2}$ State Key Laboratory of Plant Genomics and National Center For Plant Gene Research, Institute of Genetics and Developmental Biology, Chinese Academy of Sciences, NO.1 Beichen West Road, Chaoyang District, Beijing 100101, China. ${ }^{3}$ University of Chinese Academy of Sciences, No.19(A) Yuquan Road, Shijingshan District, Beijing 100049, China.

Received: 30 March 2018 Accepted: 1 August 2018

Published online: 06 August 2018

\section{References}

1. Hori K, Matsubara K, Yano M. Genetic control of flowering time in rice: integration of Mendelian genetics and genomics. Theor Appl Genet. 2016; 129(12):2241-52.

2. Izawa T. Adaptation of flowering-time by natural and artificial selection in Arabidopsis and rice. J Exp Bot. 2007:58(12):3091-7.

3. Ikeda M, Miura K, Aya K, Kitano H, Matsuoka M. Genes offering the potential for designing yield-related traits in rice. Curr Opin Plant Biol. 2013;16(2):213-20.

4. Hayama R, Izawa T, Shimamoto K. Isolation of rice genes possibly involved in the photoperiodic control of flowering by a fluorescent differential display method. Plant Cell Physiol. 2002;43(5):494-504.

5. Yano M, Katayose $Y$, Ashikari M, Yamanouchi U, Monna L, Fuse T, Baba T, Yamamoto K, Umehara Y, Nagamura Y, et al. Hd1, a major photoperiod sensitivity quantitative trait locus in rice, is closely related to the Arabidopsis flowering time gene CONSTANS. Plant Cell. 2000;12(12):2473-84.

6. Kojima S, Takahashi Y, Kobayashi Y, Monna L, Sasaki T, Araki T, Yano M. $\mathrm{Hd} 3 \mathrm{a}$, a rice ortholog of the Arabidopsis FT gene, promotes transition to flowering downstream of Hd1 under short-day conditions. Plant Cell Physiol. 2002:43(10):1096-105.

7. Hayama R, Yokoi S, Tamaki S, Yano M, Shimamoto K. Adaptation of photoperiodic control pathways produces short-day flowering in rice. Nature. 2003;422(6933):719-22. 
8. Doi K, Izawa T, Fuse T, Yamanouchi U, Kubo T, Shimatani Z, Yano M, Yoshimura A. Ehd1, a B-type response regulator in rice, confers short-day promotion of flowering and controls FT-like gene expression independently of Hd1. Genes Dev. 2004;18(8):926-36.

9. Xue W, Xing Y, Weng X, Zhao Y, Tang W, Wang L, Zhou H, Yu S, Xu C, Li X, et al. Natural variation in Ghd7 is an important regulator of heading date and yield potential in rice. Nat Genet. 2008;40(6):761-7.

10. Komiya R, Yokoi S, Shimamoto K. A gene network for long-day flowering activates RFT1 encoding a mobile flowering signal in rice. Development. 2009;136(20):3443-50.

11. Komiya R, Ikegami A, Tamaki S, Yokoi S, Shimamoto K. Hd3a and RFT1 are essential for flowering in rice. Development. 2008;135(4):767-74.

12. Tamaki S, Matsuo S, Wong HL, Yokoi S, Shimamoto K. Hd3a protein is a mobile flowering signal in rice. Science. 2007;316(5827):1033-6.

13. Ishikawa R, Aoki M, Kurotani K, Yokoi S, Shinomura T, Takano M, Shimamoto K. Phytochrome B regulates heading date 1 ( $\mathrm{Hd} 1)$-mediated expression of rice florigen Hd3a and critical day length in rice. Mol Gen Genomics. 2011 285(6):461-70.

14. Matsubara K, Yamanouchi U, Wang ZX, Minobe Y, Izawa T, Yano M. Ehd2, a rice ortholog of the maize INDETERMINATE1 gene, promotes flowering by up-regulating Ehd1. Plant Physiol. 2008;148(3):1425-35.

15. Matsubara K, Yamanouchi U, Nonoue $Y$, Sugimoto K, Wang ZX, Minobe $Y$, Yano M. Ehd3, encoding a plant homeodomain finger-containing protein, is a critical promoter of rice flowering. Plant J. 2011;66(4):603-12.

16. Gao H, Zheng XM, Fei G, Chen J, Jin M, Ren Y, Wu W, Zhou K, Sheng P, Zhou F, et al. Ehd4 encodes a novel and Oryza-genus-specific regulator of photoperiodic flowering in rice. PLoS Genet. 2013;9(2):e1003281.

17. Kim SL, Lee S, Kim HJ, Nam HG, An G. OsMADS51 is a short-day flowering promoter that functions upstream of Ehd1, OsMADS14, and Hd3a. Plant Physiol. 2007;145(4):1484-94.

18. Lee YS, Jeong DH, Lee DY, Yi J, Ryu CH, Kim SL, Jeong HJ, Choi SC, Jin P, Yang J, et al. OsCOL4 is a constitutive flowering repressor upstream of Ehd1 and downstream of OsphyB. Plant J. 2010;63(1):18-30.

19. Tan J, Jin M, Wang J, Wu F, Sheng P, Cheng Z, Wang J, Zheng X, Chen L, Wang $M$, et al. OsCOL10, a CONSTANS-like gene, functions as a flowering time repressor downstream of Ghd7 in Rice. Plant Cell Physiol. 2016;57(4): 798-812.

20. Wei X, Xu J, Guo H, Jiang L, Chen S, Yu C, Zhou Z, Hu P, Zhai H, Wan J. DTH8 suppresses flowering in rice, influencing plant height and yield potential simultaneously. Plant Physiol. 2010;153(4):1747-58.

21. Lin X, Wu F, Du X, Shi X, Liu Y, Liu S, Hu Y, Theissen G, Meng Z. The pleiotropic SEPALLATA-like gene OsMADS34 reveals that the 'empty glumes' of rice (Oryza sativa) spikelets are in fact rudimentary lemmas. New Phytol. 2014;202(2):689-702.

22. Choi SC, Lee S, Kim SR, Lee YS, Liu C, Cao X, An G. Trithorax group protein Oryza sativa Trithorax 1 controls flowering time in rice via interaction with early heading date3. Plant Physiol. 2014;164(3):1326-37.

23. Wang K, Tang D, Hong L, Xu W, Huang J, Li M, Gu M, Xue Y, Cheng Z. DEP and AFO regulate reproductive habit in rice. PLoS Genet. 2010;6(1):e1000818.

24. Lee S, Kim J, Han JJ, Han MJ, An G. Functional analyses of the flowering time gene OsMADS50, the putative SUPPRESSOR OF OVEREXPRESSION OF CO 1/ AGAMOUS-LIKE 20 (SOC1/AGL20) ortholog in rice. Plant J. 2004;38(5):754-64.

25. Ryu CH, Lee S, Cho LH, Kim SL, Lee YS, Choi SC, Jeong HJ, Yi J, Park SJ, Han CD, et al. OsMADS50 and OsMADS56 function antagonistically in regulating long day (LD)-dependent flowering in rice. Plant Cell Environ. 2009;32(10):1412-27.

26. Wang J, Wu F, Zhu S, Xu Y, Cheng Z, Wang J, Li C, Sheng P, Zhang H, Cai $M$, et al. Overexpression of OsMYB1R1-VP64 fusion protein increases grain yield in rice by delaying flowering time. FEBS Lett. 2016;590(19):3385-96.

27. Bai B, Zhao J, Li Y, Zhang F, Zhou J, Chen F, Xie X. OsBBX14 delays heading date by repressing florigen gene expression under long and short-day conditions in rice. Plant Sci. 2016;247:25-34.

28. Sheng P, Wu F, Tan J, Zhang H, Ma W, Chen L, Wang J, Wang J, Zhu S, Guo $X$, et al. A CONSTANS-like transcriptional activator, OsCOL13, functions as a negative regulator of flowering downstream of OsphyB and upstream of Ehd1 in rice. Plant Mol Biol. 2016;92(1-2):209-22.

29. Ambavaram MM, Basu S, Krishnan A, Ramegowda V, Batlang U, Rahman L, Baisakh N, Pereira A. Coordinated regulation of photosynthesis in rice increases yield and tolerance to environmental stress. Nat Commun. 2014;5:5302.

30. Fu FF, Xue HW. Coexpression analysis identifies Rice starch Regulator1, a rice AP2/EREBP family transcription factor, as a novel rice starch biosynthesis regulator. Plant Physiol. 2010;154(2):927-38.
31. Song $\mathrm{YH}$, Ito $\mathrm{S}$, Imaizumi T. Flowering time regulation: photoperiod- and temperature-sensing in leaves. Trends Plant Sci. 2013;18(10):575-83.

32. Song YH, Shim JS, Kinmonth-Schultz HA, Imaizumi T. Photoperiodic flowering: time measurement mechanisms in leaves. Annu Rev Plant Biol. 2014;66:441-64.

33. Wang L, Czedik-Eysenberg A, Mertz RA, Si Y, Tohge T, Nunes-Nesi A, Arrivault S, Dedow LK, Bryant DW, Zhou W, et al. Comparative analyses of C (4) and C (3) photosynthesis in developing leaves of maize and rice. Nat Biotechnol. 2014;32(11):1158-65.

34. Aoki K, Ogata Y, Shibata D. Approaches for extracting practical information from gene co-expression networks in plant biology. Plant Cell Physiol. 2007; 48(3):381-90.

35. Hirai MY, Sugiyama K, Sawada Y, Tohge T, Obayashi T, Suzuki A, Araki R, Sakurai N, Suzuki H, Aoki K, et al. Omics-based identification of Arabidopsis Myb transcription factors regulating aliphatic glucosinolate biosynthesis. Proc Natl Acad Sci U S A. 2007;104(15):6478-83.

36. Wang $Y$, Yu H, Tian C, Sajjad M, Gao C, Tong Y, Wang X, Jiao Y. Transcriptome association identifies regulators of wheat spike architecture. Plant Physiol. 2017;175(2):746-57.

37. Mounet F, Moing A, Garcia V, Petit J, Maucourt M, Deborde C, Bernillon S, Le Gall G, Colquhoun I, Defernez M, et al. Gene and metabolite regulatory network analysis of early developing fruit tissues highlights new candidate genes for the control of tomato fruit composition and development. Plant Physiol. 2009;149(3):1505-28.

38. Lu X, Li QT, Xiong Q, Li W, Bi YD, Lai YC, Liu XL, Man WQ, Zhang WK, Ma B, et al. The transcriptomic signature of developing soybean seeds reveals the genetic basis of seed trait adaptation during domestication. Plant J. 2016; 86(6):530-44.

39. Safi A, Medici A, Szponarski W, Ruffel S, Lacombe B, Krouk G. The world according to GARP transcription factors. Curr Opin Plant Biol. 2017;39:159-67.

40. Bian XF, Liu X, Zhao ZG, Jiang L, Gao H, Zhang YH, Zheng M, Chen LM, Liu SJ, Zhai $\mathrm{HQ}$, et al. Heading date gene, dth3 controlled late flowering in $\mathrm{O}$. Glaberrima Steud. By down-regulating Ehd1. Plant Cell Rep. 2011;30(12):2243-54.

41. Hori K, Ogiso-Tanaka E, Matsubara K, Yamanouchi U, Ebana K, Yano M. Hd16, a gene for casein kinase I, is involved in the control of rice flowering time by modulating the day-length response. Plant J. 2013;76(1):36-46.

42. Peng LT, Shi ZY, Li L, Shen GZ, Zhang JL. Overexpression of transcription factor OsLFL1 delays flowering time in Oryza sativa. J Plant Physiol. 2008; 165(8):876-85.

43. Matsubara K, Ogiso-Tanaka E, Hori K, Ebana K, Ando T, Yano M. Natural variation in Hd17, a homolog of Arabidopsis ELF3 that is involved in rice photoperiodic flowering. Plant Cell Physiol. 2012;53(4):709-16.

44. Koo BH, Yoo SC, Park JW, Kwon CT, Lee BD, An G, Zhang Z, Li J, Li Z, Paek NC. Natural variation in OsPRR37 regulates heading date and contributes to rice cultivation at a wide range of latitudes. Mol Plant. 2013;6(6):1877-88.

45. Li P, Ponnala L, Gandotra N, Wang L, Si Y, Tausta SL, Kebrom TH, Provart N, Patel R, Myers CR, et al. The developmental dynamics of the maize leaf transcriptome. Nat Genet. 2010;42(12):1060-7.

46. Jin J, Zhang $H$, Kong L, Gao G, Luo J. PlantTFDB 3.0: a portal for the functional and evolutionary study of plant transcription factors. Nucleic Acids Res. 2014;42(Database issue):D1182-7.

47. Shannon P, Markiel A, Ozier O, Baliga NS, Wang JT, Ramage D, Amin N, Schwikowski B, Ideker T. Cytoscape: a software environment for integrated models of biomolecular interaction networks. Genome Res. 2003;13(11):2498-504.

48. Thompson JD, Gibson TJ, Higgins DG. Multiple sequence alignment using ClustalW and ClustalX. Current protocols in bioinformatics. 2002; Chapter 2:Unit 23

49. Kumar S, Stecher G, Tamura K. MEGA7: molecular evolutionary genetics analysis version 7.0 for bigger datasets. Mol Biol Evol. 2016;33(7):1870-4.

50. Finn RD, Coggill P, Eberhardt RY, Eddy SR, Mistry J, Mitchell AL, Potter SC, Punta M, Qureshi M, Sangrador-Vegas A, et al. The Pfam protein families database: towards a more sustainable future. Nucleic Acids Res. 2016;44(D1): D279-85.

51. Hiei Y, Ohta S, Komari T, Kumashiro T. Efficient transformation of rice (Oryza sativa L.) mediated by agrobacterium and sequence analysis of the boundaries of the T-DNA. Plant J. 1994;6(2):271-82.

52. Lu Y, Ye X, Guo R, Huang J, Wang W, Tang J, Tan L, Zhu JK, Chu C, Qian Y. Genome-wide targeted mutagenesis in Rice using the CRISPR/Cas9 system. Mol Plant. 2017:10(9):1242-5.

53. Bart R, Chern M, Park CJ, Bartley L, Ronald PC. A novel system for gene silencing using siRNAs in rice leaf and stem-derived protoplasts. Plant Methods. 2006;2:13. 
54. Yang Y, Fu D, Zhu C, He Y, Zhang H, Liu T, Li X, Wu C. The RING-finger ubiquitin ligase HAF1 mediates heading date 1 degradation during photoperiodic flowering in Rice. Plant Cell. 2015;27(9):2455-68.

55. Yan WH, Wang $P$, Chen HX, Zhou HJ, Li QP, Wang CR, Ding ZH, Zhang YS, Yu SB, Xing YZ, et al. A major QTL, Ghd8, plays pleiotropic roles in regulating grain productivity, plant height, and heading date in rice. Mol Plant. 2011;4(2):319-30

Ready to submit your research? Choose BMC and benefit from:

- fast, convenient online submission

- thorough peer review by experienced researchers in your field

- rapid publication on acceptance

- support for research data, including large and complex data types

- gold Open Access which fosters wider collaboration and increased citations

- maximum visibility for your research: over $100 \mathrm{M}$ website views per year

At BMC, research is always in progress.

Learn more biomedcentral.com/submissions 